\title{
Endoplasmic Reticulum Stress Inducer Tunicamycin Alters Hepatic Energy Homeostasis in Mice
}

\author{
Bin Feng ${ }^{1,2, *,+} \mathbb{D}^{\mathbb{D}}$, Xiaohua Huang ${ }^{1,2,+}$, Dandan Jiang ${ }^{1}$, Lun Hua ${ }^{1}$, Yong Zhuo ${ }^{1}$ and De Wu ${ }^{1,2, *}$ \\ 1 Animal Nutrition Institute, Sichuan Agricultural University, Chengdu 611130, China; \\ hxh3028@163.com (X.H.); dandanjiang@163.com (D.J.); hualun0516@163.com (L.H.); \\ zhuoyong2003@163.com (Y.Z.) \\ 2 Key Laboratory of Animal Disease-Resistant Nutrition of Ministry of Education, \\ Sichuan Agricultural University, Chengdu 611130, China \\ * Correspondence: fengbin@sicau.edu.cn (B.F.); wude@sicau.edu.cn (D.W.); \\ Tel.: +86-28-8629-0922 (B.F.); +86-28-8629-0990 (D.W.) \\ + These authors contributed equally to this work.
}

Received: 22 July 2017; Accepted: 3 August 2017; Published: 4 August 2017

\begin{abstract}
Disorders of hepatic energy metabolism, which can be regulated by endoplasmic reticulum (ER) stress, lead to metabolic diseases such as hepatic steatosis and hypoglycemia. Tunicamycin, a pharmacological ER stress inducer, is used to develop an anti-cancer drug. However, the effects of tunicamycin on hepatic energy metabolism have not been well elucidated. Mice were intraperitoneally injected with tunicamycin or vehicle. Twenty-four hours later, hepatic triglyceride and glycogen content and serum lipids profiles were analyzed, as well as the expression of lipogenic and gluconeogenic genes. Tunicamycin significantly induced hepatic a yellowish color and ER stress, as well as increasing serum levels of aspartate transaminase and alanine transaminase. Besides, tunicamycin remarkably increased hepatic triglyceride content and suppressed the expression of apolipoprotein B100. In addition, tunicamycin-treated mice had lower serum levels of triglyceride, apolipoprotein B, low-density lipoprotein cholesterol and high-density lipoprotein cholesterol. Gene expression of peroxisome proliferator-activated receptor $\alpha$ was decreased by tunicamycin, but the protein level was increased. Furthermore, blood glucose level and hepatic glycogen content were decreased in tunicamycin-treated mice. Protein kinase B signaling was attenuated in the tunicamycin-treated liver, but the expression and activities of phosphoenolpyruvate carboxykinase and glucose-6-phosphatase were unchanged. Tunicamycin alters hepatic energy homeostasis by increasing triglyceride accumulation and decreasing glycogen content.
\end{abstract}

Keywords: tunicamycin; liver; triglyceride; lipoprotein; glycogen; blood glucose; ER stress; Akt

\section{Introduction}

Liver, the primary metabolic organ, plays an important role in the systemic energy homeostasis, including glucose production, lipogenesis, fatty acid oxidation, lipoprotein secretion and glycogen synthesis [1]. To be specific, firstly, the liver regulates blood glucose homeostasis by stimulating gluconeogenesis, a process mediated by phosphoenolpyruvate carboxykinase (PEPCK) and glucose 6-phosphatase (G6pase), whose expression are mainly regulated by insulin signaling and endoplasmic reticulum (ER) stress [2-4]. Secondly, the liver is one of the leading organs for lipids metabolism, including lipogenesis, cholesterol synthesis and fatty acid oxidation [5]. In the liver, lipogenesis is regulated by enzymes like fatty acid synthase (FAS) and stearoyl-CoA desaturase 1 (SCD1), while fatty acid oxidation is controlled by enzymes such as carnitine palmitoyltransferase 1a (CPT1a) and acyl-CoA dehydrogenase, long chain (ACADL) [6,7]. Stimulation of lipogenesis or inhibition of fatty acid oxidation in the liver leads to hepatic triglyceride accumulation, which results in hepatic 
steatosis [8]. In addition, reduced apolipoprotein expression and impaired hepatic lipoprotein secretion give rise to liver triglyceride accumulation [9]. Thirdly, liver synthesizes and stores glycogen, which can be broken down at fasting state to provide glucose to keep blood glucose homeostasis [10]. Synthesis of glycogen is regulated by enzyme of glycogen synthetase (GS) while glycogen breakdown is mediated by glycogen phosphotase (GP), both of which are regulated by insulin signaling [10,11]. Moreover, impaired liver glycogen accumulation leads to hypoglycemia under fasting state [10].

In the liver, ER stress restores ER homeostasis by stimulating the activation of inositol requiring enzyme 1 (IRE1), activating transcription factor 6 (ATF6) and RNA-dependent protein kinase-like ER kinase (PERK), which also regulate energy metabolism [4]. Firstly, IRE1 activation prevents the inhibition of insulin signaling on forkhead box O1 (FOXO1) activation, thus induces gluconeogenesis, which is known as insulin resistance [4]. Secondly, PERK activation stimulates lipogenesis and gluconeogenesis by inducing the expression of CHOP through eukaryotic initiation factor $2 \alpha$ (eIF2 $\alpha$ )/ATF4 pathway [12]. Thirdly, ER stress induces the expression of the nuclear form of ATF6, which inhibits gluconeogenesis and lipogenesis by binding to and inactivating CREB-regulated transcription coactivator 2 (TORC2) and sterol-regulatory element binding protein 2 (SREBP2), respectively $[13,14]$. Lastly, ER stress stimulates the splicing of X-box binding protein 1 (Xbp1), which can directly or indirectly (through SREBP1) activate lipogenesis program while inhibiting gluconeogenesis $[15,16]$. ER stress is induced by unfolded protein response (UPR), which can be stimulated by some chemicals [3].

Tunicamycin, a pharmacological ER stress inducer, can stimulate tumor cell apoptosis $[17,18]$. Therefore, it has been used to develop an anti-cancer drug [19,20]. Because of its ER stress inducing character, tunicamycin has been reported to induce metabolism disorders in many studies. As is demonstrated in a study on hepatocytes, tunicamycin inhibits the phosphorylation of protein kinase B (Akt) [21], which plays a vital role in insulin sensitivity and in the regulation of glucose and triglyceride metabolism [22]. Wang et al. suggested that short-time tunicamycin treatment inhibited hepatic gluconeogenesis [13]. Furthermore, tunicamycin was revealed in a recent study to induce triglyceride accumulation in cultured HepG2 hepatic cells [23]. Chang and his colleagues demonstrated that short-term tunicamycin treatment increased hepatic lipid accumulation [24]. However, the in vivo effects of tunicamycin on hepatic gluconeogenesis, triglyceride accumulation and glycogen synthesis, and the exact mechanism have not been fully illustrated yet.

In the current study, the effects of $24 \mathrm{~h}$ tunicamycin treatment on serum lipids profiles, hepatic triglyceride accumulation, blood glucose level and liver glycogen content were explored using a mouse model. Meanwhile, the possible regulatory mechanism was investigated. Results indicated that, as compared with the control group, $24 \mathrm{~h}$ tunicamycin exposure dramatically increased hepatic triglyceride accumulation, inhibited liver lipoprotein secretion, decreased blood glucose level and hepatic glycogen content.

\section{Results}

\subsection{Tunicamycin Induced Endoplasmic Reticulum Stress}

To investigate the effect of tunicamycin on liver energy homeostasis, mice were administrated with tunicamycin or vehicle for $24 \mathrm{~h}$ and were harvested under fed state. Result showed that tunicamycin did not alter the body weight when compared with the control group (Figure S1). However, the liver turned yellow, and the weight tended to decrease in tunicamycin-treated mice as compared to that of the control mice (Figure 1A,B). As tunicamycin is a pharmacologic ER stress inducer, the ER stress levels were detected in the liver. Results showed that, when compared with the control group, the phosphorylation level of PERK, an indicator of ER stress [3], was greatly induced in tunicamycin-treated liver (Figure 1C). In addition, tunicamycin up-regulated the expression of Chop and $78 \mathrm{kDa}$ glucose-regulated protein (Grp78) (Figure 1D), two indicator genes of ER stress [25,26]. These data indicated that $24 \mathrm{~h}$ tunicamycin administration induced ER stress in the liver. In addition, 
the serum aspartate transaminase (AST) and alanine transaminase (ALT) levels were detected to reflect the liver function, which showed that the serum levels of both AST and ALT were higher in tunicamycin treated mice than these in the control mice (Figure 1E,F). However, the ratio of AST to ALT was unchanged (Figure 1G). These data indicated that tunicamycin slightly impaired liver function.

$\mathbf{A}$

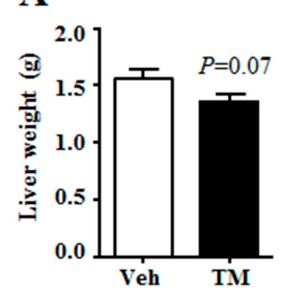

B

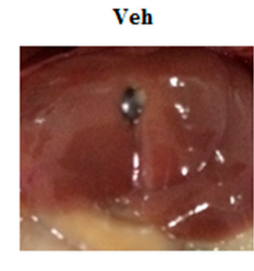

C PERK

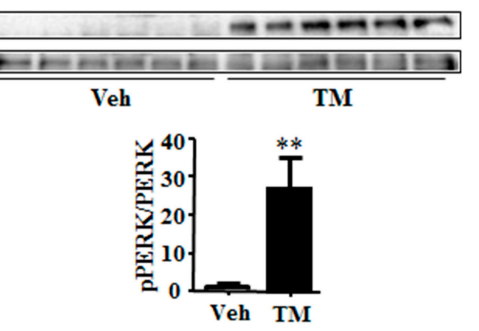

D

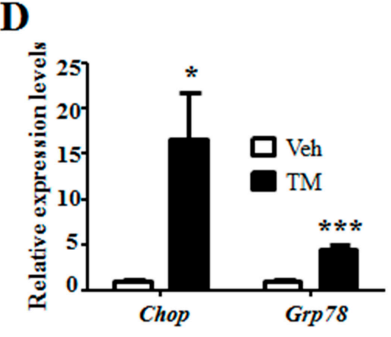

$\mathbf{E}$

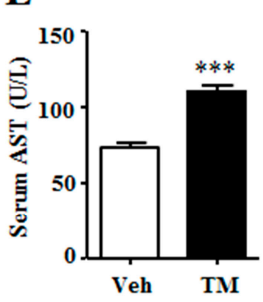

F

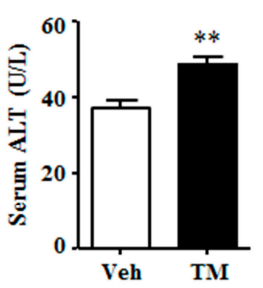

G

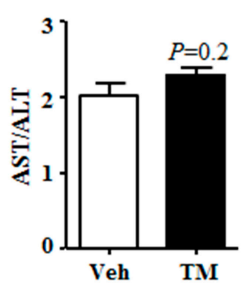

Figure 1. Tunicamycin induced hepatic endoplasmic reticulum (ER) stress and impaired liver function. 7-month-old mice were injected intraperitoneally with vehicle or $1 \mathrm{mg} / \mathrm{kg}$ tunicamycin. Livers were collected $24 \mathrm{~h}$ after injection under fed condition. $N=6$ per group. (A) Liver weight at harvest; (B) Liver morphology; (C) Phosphorylation level of PERK in the liver; (D) Expression levels of ER stress indicator genes in the liver; (E) Serum level of AST; (F) Serum level of ALT; (G) The ratio of serum AST to ALT. Data were shown as mean \pm SEM. Veh, vehicle; TM, tunicamycin. ${ }^{*} p<0.05,{ }^{* *} p<0.01,{ }^{* * *} p<0.001$ TM vs. Veh.

\subsection{Tunicamycin Administration Decreased Blood Glucose Level}

It is reported that ER stress regulates hepatic gluconeogenesis [4], thus the blood glucose level was measured. Results indicated that, tunicamycin-treated mice had lower blood glucose levels as compared to the control mice (Figure 2A). In addition, insulin tolerance test revealed that tunicamycin-treated mice were more sensitivity to insulin than the control ones (Figure 2B). Nonetheless, tunicamycin did not affect the ability of glucose tolerance in mice (Figure S1B). In addition, serum insulin levels in tunicamycin-treated mice were similar to the control mice (Figure S1C). Gluconeogenesis plays an important role in systemic glucose homeostasis, while PEPCK and G6pase are the two rate-limiting enzymes for gluconeogenesis [2]. The results showed that tunicamycin up-regulated the expression of Pepck while G6pase expression remained unaffected (Figure 2C). Protein levels of PEPCK and G6Pase were also determined, which indicated that the two in tunicamycin-treated liver were not different from those in control liver (Figure 2D). Further study revealed no significant difference in the activity of PEPCK and G6Pase between tunicamycin group and control group (Figure 2E,F). Besides, liver content of pyruvate, the substrate of gluconeogenesis, trended to be higher in tunicamycin-treated mice than that in the control mice (Figure S1D). These findings demonstrated that $24 \mathrm{~h}$ tunicamycin treatment decreased blood glucose but it did not affect liver gluconeogenesis. 
A

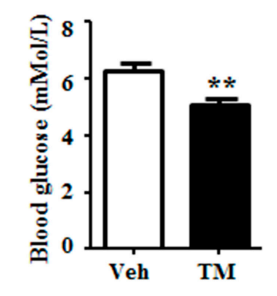

C

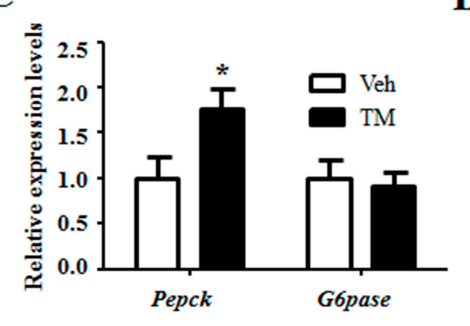

B

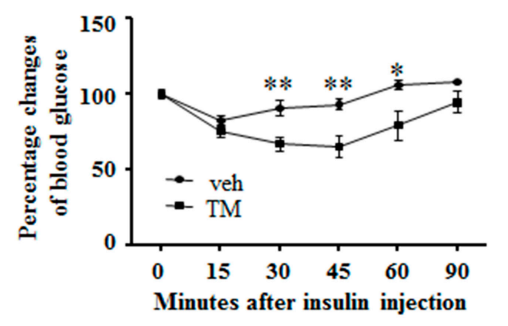

D

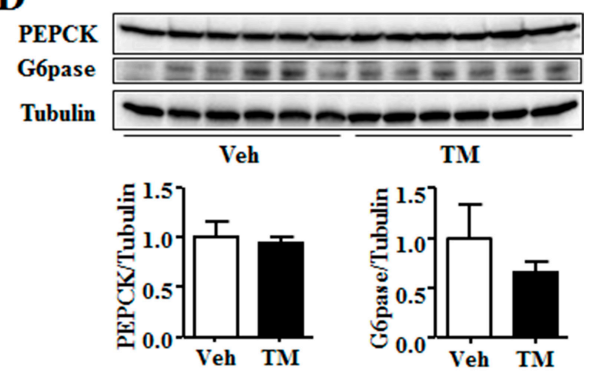

$\mathbf{E}$

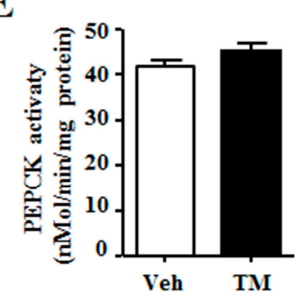

$\mathbf{F}$

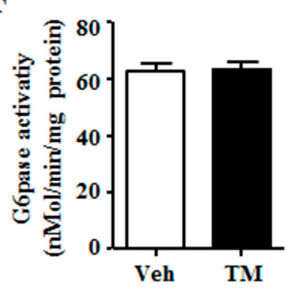

Figure 2. Tunicamycin reduced blood glucose level. (A) Blood glucose levels under fed state; $N=6$ per group; (B) Insulin tolerance test. $N=7$ per group; (C) Expression of gluconeogenic genes. $N=6$ per group; (D) Western blotting bands and quantification for liver proteins; (E) The enzyme activity of PEPCK; (F) The enzyme activity of G6pase. Veh, vehcle; TM, tunicamycin. ${ }^{*} p<0.05,{ }^{* *} p<0.01 \mathrm{TM}$ vs. Veh.

\subsection{Tunicamycin Induced Hepatic Triglyceride Accumulation}

The altered liver weight indicated that the content of tunicamycin-treated liver might be different from the content of control liver. Thus, the triglyceride and glycogen contents were detected in the liver. Results showed that tunicamycin remarkably increased the content of hepatic triglyceride (Figure 3A), an important energy source in the liver [27]. Meanwhile, H\&E staining and Oil Red O staining also revealed that livers from tunicamycin treated mice had accumulated more and larger lipid droplets than those from control ones (Figure 3B,C). These data suggested that $24 \mathrm{~h}$ tunicamycin administration was susceptible to induce hepatic steatosis.

\subsection{Tunicamycin Inhibited Hepatic Lipoprotein Secretion}

ER stress has been reported to stimulate lipogenesis [4]. Thus, the expression levels of lipogenic genes were measured. The results indicated that, the expression of Fas and Scd1 were notably down-regulated by tunicamycin rather than being up-regulated (Figure 4A). This result was supported by the decreased protein levels of FAS and SCD1 in the tunicamycin treated liver (Figure 4B). Consequently, the expression of fatty acid oxidation related genes was thus detected. Results showed that tunicamycin inhibited the gene expression of Ppar, a transcription stimulator of fatty acid oxidation genes (Figure 4C). However, the protein level of PPAR $\alpha$ was significantly increased in tunicamycin-treated liver as compared to that in the control liver (Figure 4B). Meanwhile, the gene expression level of $\mathrm{Cpt1a}$, an important fatty acid oxidation related gene, was increased in the tunicamycin-treated liver (Figure 4D). Furthermore, though the gene expression level of Acadl, another 
fatty acid oxidation gene, was decreased in tunicamycin-treated liver as compared to that in the control liver, the protein level was unchanged (Figure 4B,D). Besides, the content of acetyl CoA, the substrate for lipogenesis and production of fatty acid oxidation, was higher in tunicamycin-treated liver than this in the control liver (Figure 4E). These data suggested that the increased triglyceride content in $24 \mathrm{~h}$ tunicamycin-treated liver was probably independent on lipogenesis and fatty acid oxidation.

A

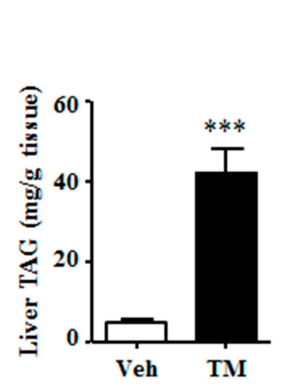

B

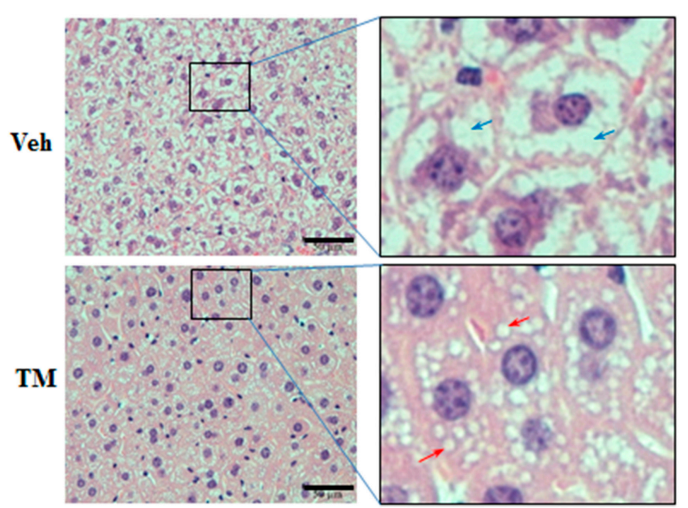

C

Veh

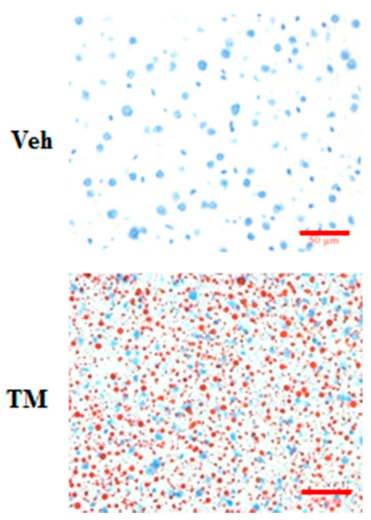

Figure 3. Tunicamycin induced liver triglyceride accumulation. 7-month-old mice were intraperitoneally injected with vehicle or $1 \mathrm{mg} / \mathrm{kg}$ tunicamycin. Livers were collected $24 \mathrm{~h}$ after injection. $N=6$ per group. (A) Liver triglyceride content; (B) H\&E staining of liver. Red arrows indicated lipid droplets, and blue arrows stood for glycogen particles; (C) Oil-red O staining of liver. Scale bars were equal to $50 \mu \mathrm{m}$. Data were expressed as mean \pm SEM. Veh, vehicle; TM, tunicamycin. *** $p<0.001$ TM vs. Veh.

A

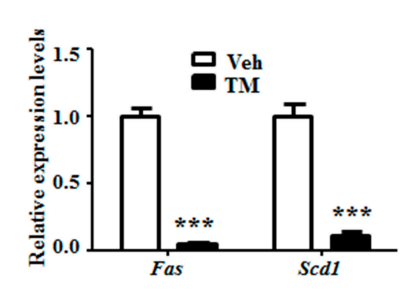

C

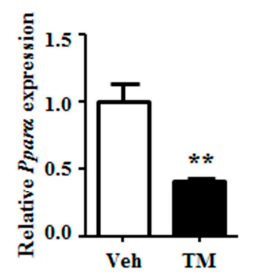

D
B
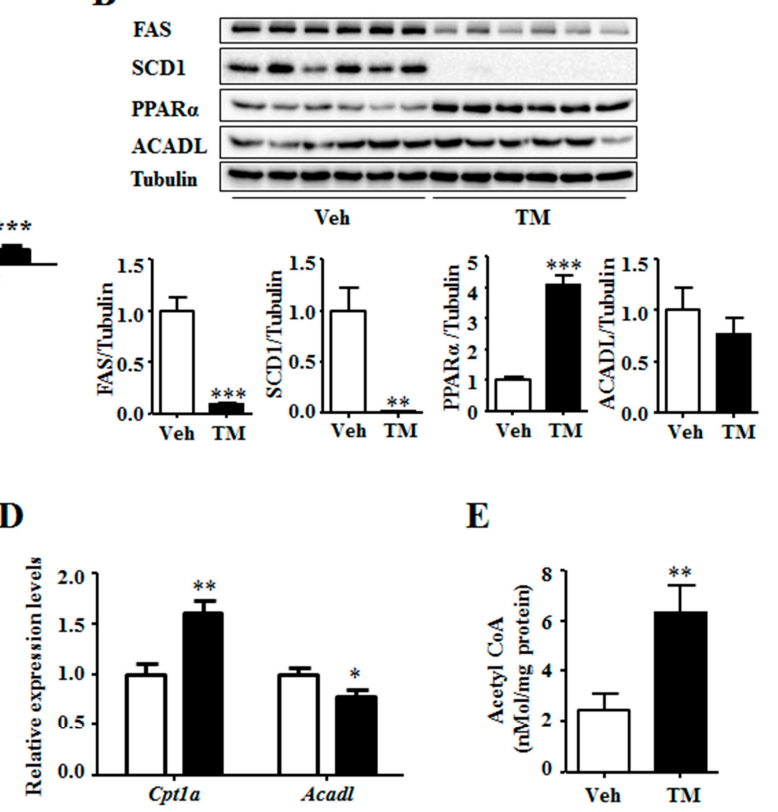

$\mathbf{E}$

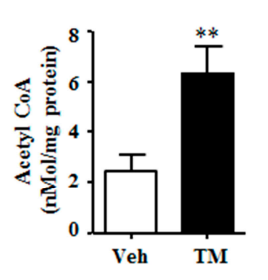

Figure 4. Tunicamycin inhibited the expression of liver lipogenic genes and increased the protein level of PPAR $\alpha$. (A) Expression of lipogenic genes; (B) Protein levels of lipogenic and fatty acid oxidation related genes; (C) Expression of fatty acid oxidation stimulator gene Ppara. (D) Expression of fatty acid oxidation related genes; (E) The acetyl CoA content in the liver. $N=6$ per group. Veh, vehcle; TM, tunicamycin. ${ }^{*} p<0.05,{ }^{* *} p<0.01,{ }^{* * *} p<0.001 \mathrm{TM}$ vs. Veh. 
The lipoprotein secretion of liver was then analyzed. Data indicated that the expression of $A p o B 100$, the major apolipoprotein of liver, was inhibited in tunicamycin-treated liver as compared to that in the control liver (Figure 5A). Meanwhile, serum levels of triglyceride, ApoB, low-density lipoprotein cholesterol (LDL-C) and high-density protein cholesterol (HDL-C) were significantly lower in tunicamycin-treated mice than those in control mice (Figure 5B-E). Besides, serum free fatty acid (FFA) level was also decreased in tunicamycin-treated mice (Figure 5F). These data indicated that tunicamycin impaired hepatic lipoprotein secretion.

A

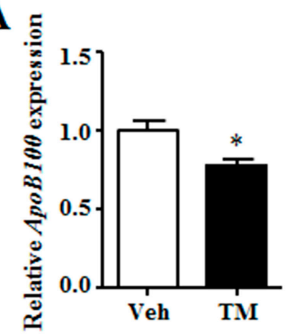

D

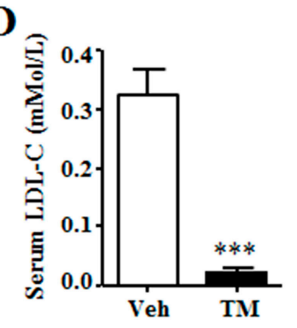

B

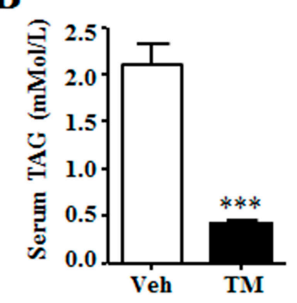

$\mathbf{E}$

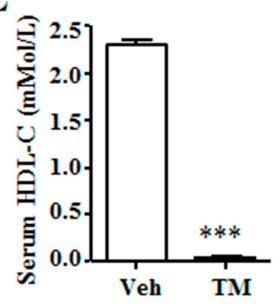

$\mathrm{C}$

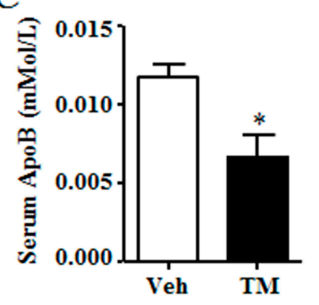

$\mathbf{F}$

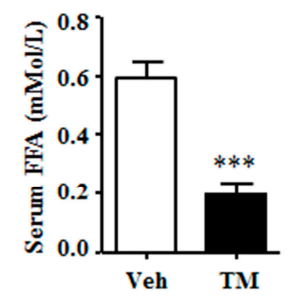

Figure 5. Tunicamycin impaired hepatic lipoprotein secretion. (A) Gene expression of ApoB100 in the liver; (B) Serum levels of triglyceride; (C) Serum levels of apolipoprotein B; (D) Serum levels of low-density lipoprotein cholesterol; (E) Serum levels of high-density lipoprotein cholesterol; (F) Serum levels of free fatty acid. $N=6$ per group. Veh, vehcle; TM, tunicamycin. ${ }^{*} p<0.05,{ }^{* * *} p<0.001 \mathrm{TM}$ vs. Veh.

\subsection{Tunicamycin Attenuated Liver Glycogen Accumulation}

The results showed that tunicamycin had decreased the content of liver glycogen (Figure 5A), another important energy source in liver besides triglyceride [10]. Morphological analysis of H\&E and PAS staining also led to similar result (Figures 2B and 5B). Akt activation stimulates liver glycogen synthesis [28]. Therefore, the phosphorylation level of Akt was investigated, the results of which showed that tunicamycin reduced the phosphorylation level of Akt (Figure 5C). However, tunicamycin did not alter the phosphorylation level or the total protein level of GS (Figure 5C), neither the protein level of GP (Figure 5C). These data suggested that glycogen content might be reduced in a GS- and GP-expression-independent manner in $24 \mathrm{~h}$ tunicamycin-treated liver.

\section{Discussion}

ER stress has dual functions, as it induces metabolic syndrome as well as inducing tumor cell apoptosis [3]. As is shown in this study, $24 \mathrm{~h}$ tunicamycin treatment notably induces hepatic ER stress and yellowish color, impairs liver function, reduces blood glucose level, stimulates hepatic triglyceride accumulation and attenuates liver glycogen storage. However, it is also observed that 24 $\mathrm{h}$ tunicamycin treatment has no effect on the expression and the activity of gluconeogenic enzymes, which disagrees with previous report, who indicates that tunicamycin inhibits the expression of Pepck and G6pase. Besides, we observe that, though the gene expression is inhibited, the protein level of PPAR $\alpha$ is significantly increased in tunicamycin treated liver, which has not been reported previously. In addition, our study demonstrates that tunicamycin decreases hepatic lipoprotein secretion by 
inhibiting the expression of ApoB100, which has not been reported previously either. Our study also demonstrates that the increased hepatic triglyceride accumulation may be due to the inhibited hepatic lipoprotein secretion.

\subsection{Tunicamycin and ER Stress}

Results in this study have shown that tunicamycin exposure has greatly induced the expression of Chop and Grp78 and the phosphorylation level of PERK (Figure 1), suggesting that $24 \mathrm{~h}$ tunicamycin treatment gives rise to remarkable ER stress. ER stress has a dominant effect on metabolic homeostasis, especially for chronic ER stress, which accounts for one of the reasons for chronic metabolic diseases [3,4,29]. Three types of ER stress have been reported, including acute ER stress, periodic ER stress and chronic ER stress, and different types of ER stress have different effects on metabolism [4]. Of them, the effects of acute and chronic ER stress on hepatic energy metabolism have been widely studied $[3,4,30]$. However, the effect of sub-acute ER stress, the transition between acute and chronic ER stress, on metabolic homeostasis remains unknown. Acute ER stress has been studied within hours while chronic ER stress within days or longer; therefore, ER stress induced by $24 \mathrm{~h}$ tunicamycin treatment in our study is considered as the transition between acute and chronic ER stress $[3,4,30]$. This may account for the reason why the effect of tunicamycin on the expression of hepatic gluconeogenic genes in our study is different from those in previous reports. Consequently, one possibility has been raised in this study that the sub-acute ER stress has different effects on hepatic energy metabolism from those of the acute and chronic ER stress. Further studies are required to explore the role of sub-acute ER stress on energy metabolism.

Besides, tunicamycin is reported to inhibit tumor growth at dose of $1 \mathrm{mg} / \mathrm{kg}$, but not at the dose of $0.5 \mathrm{mg} / \mathrm{kg}$ [19]. However, our study indicates that tunicamycin at the dose of $1 \mathrm{mg} / \mathrm{kg}$ body weight slightly impairs liver function. This suggests that when tunicamycin or other ER stress inducers are used as anti-cancer medicine, liver protection is required.

\subsection{Tunicamycin and Blood Glucose}

It is shown in the current study that $24 \mathrm{~h}$ tunicamycin treatment decreases the blood glucose level (Figure 4), which is supported by previous reports that acute tunicamycin treatment reduces blood glucose level $[13,31]$. However, $24 \mathrm{~h}$ tunicamycin treatment makes no difference to the expression of gluconeogenic genes in this study, which is different from the previous reports declaring an inhibition of gluconeogenic genes expression by acute tunicamycin treatment. One possible reason is the duration of tunicamycin treatment, which is $24 \mathrm{~h}$ in this study, compared to $10 \mathrm{~h}$ and $6 \mathrm{~h}$ in the studies by Wang et al. and Lee et al., respectively [13,31]. Moreover, our result is also different from the report demonstrating that chronic ER stress induced by long term high fat diet intake stimulates gluconeogenesis [32].

Thus, another possibility has been raised that tunicamycin induces apoptosis of some liver cells. This may lead to decreased hepatic glucose production rate in tunicamycin-treated mice. Thus, when challenged with insulin, blood glucose levels of the tunicamycin-treated mice decrease faster than these of the control mice. At the same time, the expression and activity of PEPCK and G6pase are normalized to total $\beta$-action (gene expression), total tubulin (protein expression) or total protein (enzyme activity), including which of the apoptosis cells. So, they retain similar in both groups or is higher (gene expression of Pepck) in the tunicamycin treated liver. However, the exact mechanism needs further study to be elucidated.

\subsection{Tunicamycin and Hepatic Triglyceride}

In this study, tunicamycin is found to stimulate liver triglyceride accumulation (Figure 1), which is consistent with the previous studies by Chang et al. and by Lee et al. In their study, Chang et al. reported that tunicamycin induced triglyceride accumulation in hepatocytes, while Lee et al. observed that tunicamycin increased the triglyceride content in mouse liver [24,30]. Increased hepatic triglyceride 
accumulation induces hepatic steatosis $[8,27,33]$. Thus, it is suggested from the findings of this study that prolonged tunicamycin treatment may lead to increased risk of hepatic steatosis.

Triglyceride metabolism in liver is regulated by lipogenesis and fatty acid $\beta$-oxidation [27]. Chronic ER stress is reported to induce lipogenesis in the liver [4]. However, $24 \mathrm{~h}$ tunicamycin treatment down-regulates the gene expression and protein levels of lipogenic enzymes FAS and SCD1, which are important lipogenic genes (Figure 2). This may be attributed to the sub-acute ER stress induced by 24 $\mathrm{h}$ tunicamycin exposure in our study, which has a different effect on lipogenesis from that of chronic ER stress [4]. Lee et al. declared an increased lipogenesis in tunicamycin-treated liver. However, the expression of Fas and $S c d 1$ in the cultured hepatoma cells, rather than in the liver, was shown in their study [30]. The decreased expression of lipogenic genes in $24 \mathrm{~h}$ tunicamycin-treated liver may result from the negative feedback of increased hepatic triglyceride accumulation on lipogenesis [7]. Akt activation is reported to stimulate hepatic lipogenesis [34]. Therefore, decreased Akt phosphorylation level may also be responsible for the inhibition of lipogenic gene expression by $24 \mathrm{~h}$ tunicamycin exposure in our study (Figure 3). However, more studies are required to further elucidate the precise mechanism by which tunicamycin down-regulates the expression of lipogenic genes.

Subsequently, the expression levels of fatty acid oxidation related genes are detected. The result shows that, the expression of Ppard is significantly decreased, which is in accordance with a previous report [30]. However, the protein level of PPAR $\alpha$ is significantly higher in tunicamycin-treated liver than that in the control liver. This suggests that tunicamycin has contrary effects on transcriptional and post-transcriptional expression of PPAR $\alpha$. However, the mechanism and the role need further study to be explored. In addition, the expression of Cpt1a is increased and the protein level of ACADL was unchanged by tunicamycin. Moreover, liver content of acetyl $\mathrm{CoA}$, the substrate for lipogenesis and production of fatty acid oxidation, is increased in tunicamycin treated mice. All these data demonstrate that the fatty acid oxidation is probably not decreased by tunicamycin in our study.

Liver triglyceride content also can be regulated by hepatic lipoprotein secretion [35]. Our data demonstrate that the expression of $A p o B 100$, the liver expressed VLDL binding apolipoprotein, is reduced in the tunicamycin treated liver. Together with the reduction of serum triglyceride, ApoB, LDL-C and HDL-C levels, our data suggest that the hepatic lipoprotein secretion of tunicamycin-treated mice is inhibited, which may be the reason why hepatic triglyceride accumulation is increased in tunicamycin-treated mice.

\subsection{Tunicamycin and Liver Glycogen}

Hepatic glycogen is a storage form as well as a rapid source of blood glucose, while decreased hepatic glycogen storage adds to the risk of hypoglycemia under fasting condition [36]. $24 \mathrm{~h}$ tunicamycin treatment evidently decreases hepatic glycogen content in our study (Figure 6). It suggests that tunicamycin has impaired liver glycogen accumulation, which has not been reported previously. Akt is reported to stimulate hepatic glycogen synthesis by means of stimulating GS activity [28]. A decrease of Akt phosphorylation level has been observed in this study (Figure 3). However, the phosphorylation level or the total protein level of GS remains unchanged in tunicamycin-treated mice, indicating that tunicamycin may decrease liver glycogen content in a GS-expression independent manner. Besides, tunicamycin does not change the protein level of GP as well (Figure 3), suggesting that glycogen breakdown may not be affected either. Therefore, the reduced hepatic glycogen content may result from the decreased blood glucose level in tunicamycin-treated mice. These results indicate that cancer patients may be fasting intolerance during the treatment of tunicamycin or other ER stress inducing medicines. 


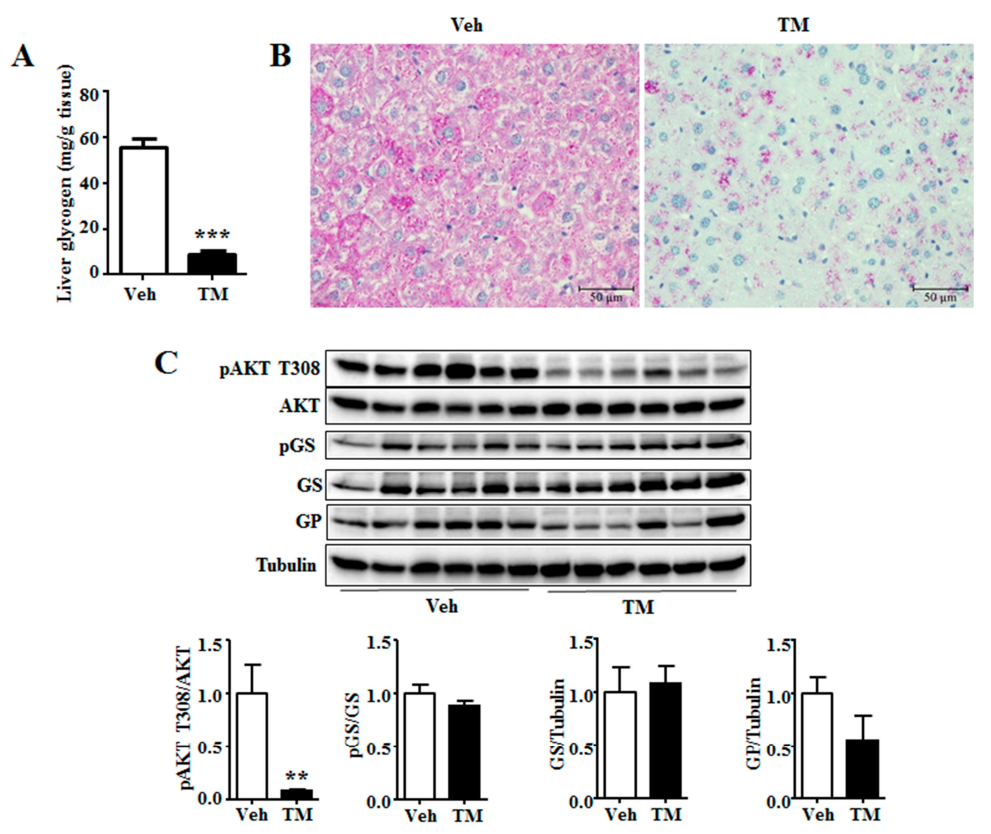

Figure 6. Tunicamycin decreased hepatic glycogen content. (A) Hepatic glycogen content; (B) PAS staining of liver; (C) Western blotting bands and quantification of liver proteins. $N=6$ per group. Scale bars were equal to $50 \mu \mathrm{m}$. Veh, vehcle; TM, tunicamycin. ${ }^{* *} p<0.01,{ }^{* * *} p<0.001 \mathrm{TM}$ vs. Veh.

Overall, $24 \mathrm{~h}$ tunicamycin exposure slightly impairs liver function, increases hepatic triglyceride accumulation, inhibits liver lipoprotein secretion and decreases blood glucose level, hepatic glycogen accumulation and serum lipids levels. Our study suggests that, chemicals, which are used to treat tumors by their ER stress inducing characteristic, may cause liver function failure and metabolic disorders, thus liver protection should be performed when these medicines are using.

\section{Materials and Methods}

\subsection{Animals and Chemicals}

Animal study protocol (MICE2015016, 10/22/2016) was reviewed and approved by the Animal Care and Use Committee of Sichuan Agricultural University. All animal procedures were performed according to the National Institutes of Health guide for the care and use of Laboratory animals. 7-month-old C57BL/ 6 male mice were obtained from Vital River Laboratory Animal Technology Co. Ltd. (Beijing, China). The mice were allowed for one week of acclimation in a pathogen-free room at the temperature of $22{ }^{\circ} \mathrm{C}$ and the humidity of $60 \%$. Subsequently, they were randomly divided into 2 groups according to similar average body weight and blood glucose level (Blood glucose strips were purchased from Beijingyicheng, Beijing, China). One group were injected intraperitoneally (IP) with $1 \mathrm{mg} / \mathrm{kg}$ tunicamycin (Sigma, Shanghai, China) or vehicle. The body weight and blood glucose level at fed state were measured $24 \mathrm{~h}$ after injection. Later, mice were euthanized using carbon dioxide, followed by cervical dislocation. Serum was collected for further analysis. Livers were rapidly dissected and weighed after taking morphological images. One piece of liver was fixed in $10 \%$ formalin for H\&E and PAS staining, while one piece was frozen in OCT embedding medium, and the remaining was frozen at $-80^{\circ} \mathrm{C}$ for further analysis.

\subsection{Insulin Tolerance Test and Glucose Tolerance Test}

For insulin tolerance test (ITT), mice were injected intraperitoneally with $1 \mathrm{mg} / \mathrm{kg}$ tunicamycin or vehicle. $18 \mathrm{~h}$ later, mice were deprived of food for $6 \mathrm{~h}$. And then, mice were injected intraperitoneally 
with $0.25 \mathrm{U} / \mathrm{kg}$ insulin (Novo Nordisk, Beijing, China). Blood glucose level was measured 0, 15, 30, 45, 60 and 90 min after insulin injection.

For glucose tolerance test (GTT), mice were intraperitoneally injected with vehicle or $1 \mathrm{mg} / \mathrm{kg}$ tunicamycin, and then were deprived of food $8 \mathrm{~h}$ after injection. $24 \mathrm{~h}$ after injection, mice were intraperitoneally injected with $1 \mathrm{~g} / \mathrm{kg}$ dextrose (Sigma). Blood glucose were measured at $0,15,30,45$, 60 and 90 min after dextrose injection.

\subsection{Serum Metabolites Profile Analysis}

Serum AST, ALT, triglyceride, ApoB, HDL-C, LDL-C and FFA levels were measured on an automatic biochemical analyzer (7020, HITACHI, Tokyo, Japan) with their analysis kits respectively (All kits were obtained from Makerbio. Co., Chengdu, China) according to the manufacturer's instructions. Serum pyruvate levels were detected using a commercial kit (Nanjing Jiancheng Bioengineering Institute, Nanjing, China) according to the manufacturer's instruction.

\subsection{Serum Insulin Level Measurement}

Serum insulin levels were measured with a mouse insulin ultrasensitive ELISA kit (ALPCO, Salem, MA, USA) according to the manufacturer's instruction.

\subsection{Liver Pyruvate and Acetyl CoA Content Measurement}

Liver pyruvate content was detected using a commercial pyruvate kit (Nanjing Jiancheng Bioengineering Institute, Nanjing, China) according to the manufacturer's instruction. Liver acetyl CoA content was detected using a commercial acetyl CoA kit (Cominbio, Suzhou, China) according to the manufacturer's instruction.

\subsection{Liver Glycogen Assay}

Liver glycogen content was measured using a commercial glycogen assay kit from Nanjing Jiancheng Bioengineering Institute according to the manufacturer's instruction with slight modification. Briefly, frozen livers were ground with a pestle in liquid nitrogen. $300 \mu \mathrm{L}$ alkaline solution was added to $100 \mathrm{mg}$ tissue powder. Mixture was heated in boiling water for $20 \mathrm{~min}$. Samples were then mixed with 96-fold (volume/tissue weight) of doubly distilled (dd) $\mathrm{H}_{2} \mathrm{O}$ and centrifuged at $5000 \times g$ for $5 \mathrm{~min}$. $200 \mu \mathrm{L}$ of the supernatant or standard was transferred to a new Eppendorf (EP) tube and mixed with $400 \mu \mathrm{L}$ chromogenic agent. The mixture was subsequently heated at $98^{\circ} \mathrm{C}$ for $5 \mathrm{~min}$. The absorbance was measured at $620 \mathrm{~nm}$ on a plate reader (SpectraMax M2, Molecular Devices, Sunnyvale, CA, USA). Liver glycogen content was calculated from the known standards multiplied by the dilution factor.

\subsection{Liver Triglycerides Assay}

Liver triglyceride content was measured using a commercial triglyceride assay kit from Nanjing Jiancheng Bioengineering Institute according to the manufacturer's instruction with modifications as previously described [37]. Briefly, $50 \mathrm{mg}$ tissue powder was homogenized in $1.5 \mathrm{~mL}$ pure ethanol with a PowerGen 125 homogenizer (Thermo Fisher Scientific, Waltham, MA, USA). The homogenate was vortexed, and then was centrifuged at $12,000 \times g$ for $5 \mathrm{~min} .1 \mathrm{~mL}$ of the supernatant was transferred to a new EP tube. $5 \mu \mathrm{L}$ samples and standards (Cat\# 17811-1AMP, Sigma) were added into a 96-well transparent plate and were incubated with $200 \mu \mathrm{L}$ enzyme mixture at $37^{\circ} \mathrm{C}$ for $10 \mathrm{~min}$. The absorbance was measured at $510 \mathrm{~nm}$ on a plate reader (Spectra Max M2). Liver triglycerides content was calculated from the known standards multiplied by the dilution factor.

\subsection{PEPCK and G6pase Activity Analysis}

The activities of hepatic PEPCK and G6pase were measured using their activity kits respectively, according to the manufacturer's instruction (Cominbio, Suzhou, China). 


\subsection{Liver Histology Staining}

For H\&E and PAS staining, fresh liver tissues were fixed in $10 \%$ formalin for $48 \mathrm{~h}$, and then dehydrated and embedded in paraffin. Embedded tissues were sliced into $4 \mu \mathrm{m}$ sections (RM2016, Leica, Shanghai, China). For H\&E staining, the sections were dehydrated, stained with hematoxylin for $5 \mathrm{~min}$, washed with $\mathrm{ddH}_{2} \mathrm{O}$, and stained with eosin for $2 \mathrm{~min}$. The sections were then dehydrated and mounted with a neutral resin onto slides. For PAS staining, the sections were dehydrated, incubated with $0.5 \%$ periodate solution for $10 \mathrm{~min}$, washed with $\mathrm{ddH}_{2} \mathrm{O}$, stained with Schiff solution for $30 \mathrm{~min}$ in a dark room, washed with $\mathrm{ddH}_{2} \mathrm{O}$, and then stained with hematoxylin for $2 \mathrm{~min}$. The sections were washed with $\mathrm{ddH}_{2} \mathrm{O}$, dehydrated, and mounted with a neutral resin onto slides.

For Oil Red O staining, fresh liver tissues were embedded in OCT embedding medium and frozen in liquid nitrogen, and then sliced into $10 \mu \mathrm{m}$ sections on a frozen section machine (Cryotome E, Thermo Fisher Scientific). The sections were dried on slides, fixed in $4 \%$ paraformaldehyde for $15 \mathrm{~min}$, washed with PBS, stained with Oil Red $\mathrm{O}$ staining solution for $1 \mathrm{~h}$, washed again with PBS, and stained with hematoxylin for $2 \mathrm{~min}$. The slides were then washed with $\mathrm{ddH}_{2} \mathrm{O}$, dried, and mounted with glycerogelatin. Images were captured on a microscope (TS100, Nikon, Tokyo, Japan) with a CCD (DS-U3, Nikon) using imaging software (NIS-Elements F3.2, Nikon).

\subsection{RNA Extraction and Real-Time PCR}

RNA extraction and real-time PCR were performed as previously reported [37]. Briefly, $50 \mathrm{mg}$ liver tissue powder was homogenized in $1 \mathrm{~mL}$ Trizol Reagent (Invitrogen, Shanghai, China) and RNA was extracted in accordance with the manufacturer's instruction. The quality of RNA was assessed by agarose gel and the concentration was measured with a spectrophotometer (NanoDrop 2000, Thermo Fisher Scientific). $1 \mu \mathrm{g}$ RNA was reverse-transcribed into cDNA with a reverse-transcription PCR kit according to the manufacturer's instructions (Takara, Dalian, China). Real-time PCR was conducted on a quantitative-PCR machine (7900HT, ABI, Carlsbad, CA, USA) with Power SYBR Green RT-PCR reagents (Thermo Fisher Scientific). The following reagent amounts were used for each reaction: forward primer, $300 \mathrm{nM}$; reverse primer, $300 \mathrm{nM}$; cDNA sample, $20 \mathrm{ng}$. The conditions used for PCR were: $95^{\circ} \mathrm{C}$ for $10 \mathrm{~min}$ for $1 \mathrm{cycle}$, and then 40 cycles of $95^{\circ} \mathrm{C}$ for $15 \mathrm{~s}$ followed by $60{ }^{\circ} \mathrm{C}$ for $1 \mathrm{~min}$. The real time PCR data was analyzed by the $2^{-\Delta \Delta C t}$ method with $\beta$-actin as the reference. The sequences of the primers are listed below:

$\beta$-actin forward GGCTGTATTCCCCTCCATCG and reverse CCAGTTGGTAACAATGCCATGT; Chop, forward CACGCACATCCCAAAGCC and reverse GGGCACTGACCACTCTGTT; Grp78, forward ATCAGGGCAACCGCATCAC and reverse TGATGTCCTGCTGCACCGAA; Pepck, forward CGCTGGATGTCGGAAGAGG and reverse GGCGAGTCTGTCAGTTCAATAC; G6pase, forward CGACTCGCTATCTCCAAGTGA and reverse GTTGAACCAGTCTCCGACCA; Fas, forward GGCTCTATGGATTACCCAAGC and reverse CCAGTGTTCGTTCCTCGGA; Scd1, forward CCTACGACAAGAACATTCAATCCC and reverse CAGGAACTCAGAAGCCCAAAGC; Ppar, forward TACTGCCGTTTTCACAAGTGC and reverse AGGTCGTGTTCACAGGTAAGA; Cpt1a, forward CTCCGCCTGAGCCATGAAG and reverse CACCAGTGATGATGCCATTCT; Acadl, forward TCTTTTCCTCGGAGCATGACA and reverse GACCTCTCTACTCACTTCTCCAG; ApoB100, forward TTGGCAAACTGCATAGCATCC and reverse TCAAATTGGGACTCTCCTTTAGC.

\subsection{Western Blot Analysis}

Western blot analysis was performed as previously reported [38]. For the preparation of protein lysates, $100 \mathrm{mg}$ liver tissue powder was homogenized in $1 \mathrm{~mL}$ cell lysis buffer (Beyotime Biotechnology, Shanghai, China) supplemented with protease inhibitor cocktail (Roche, Mannheim, Germany) on a homogenizer. The protein lysate was centrifuged at $12,000 \times g$ and $4{ }^{\circ} \mathrm{C}$ for $30 \mathrm{~min}$, and the supernatant was transferred to a new EP tube. The concentration of protein in the supernatant was measured with a BCA Protein Assay Kit (Thermo Fisher Scientific). $100 \mu \mathrm{g}$ protein was used to prepare an 
electrophoresis sample with loading buffer (BioRad, Shanghai, China) in a volume of $30 \mu \mathrm{L}$ for each sample. Proteins were separated on $12 \%$ polyacrylamide gel, and then transferred onto PVDF membranes (BioRad). The membranes were blocked in $1 \%$ BSA $/ 1 \times$ TBST for $1 \mathrm{~h}$ at room temperature, followed by incubation with the appropriate primary antibodies $(1 \mathrm{ug} / \mathrm{ml})$ overnight. ACADL antibody was the product of Abcam (Shanghai, China); pAKT T308 (4056), AKT (9272), pGS (3891), GS (3886), pPERK (3179), PERK (5683), SCD1 (2794) and tubulin (3873) antibodies were obtained from Cell Signaling Technology (Shanghai, China); and GP (sc-46347), G6pase (sc-398155), PEPCK (sc-377136), FAS (sc-48357) and PPAR $\alpha$ (sc-398394) antibodies were obtained from Santa Cruz (Shanghai, China). After thorough washing, membranes were incubated with appropriate horseradish peroxidase-linked secondary antibodies (7074 and 7076, CST) (1:2000 dilution in 5\% milk/1× TBST) for $1 \mathrm{~h}$. After further thorough washing, protein signals were detected by ECL western blotting detection reagent (BioRad, Shanghai, China) on a Molecular Imager ChemiDoc XRS+ System (BioRad, Shanghai, China). Blots were quantified with ImageJ software (National Institutes of Health, Bethesda, MD, USA).

\subsection{Statistical Analysis}

Data were analyzed with Microsoft Office Excel 2016 and Prism 5, and the results were presented as mean \pm SEM. Student's $t$-test was used to compare the difference between two groups. Statistical significance was determined at $p<0.05$.

Supplementary Materials: Supplementary materials can be found at www.mdpi.com/1422-0067/18/8/1710/s1.

Acknowledgments: Many thanks to Zhengfeng Fang, Liangqiang Che, Yan Lin, Shengyu Xu, Jian Li, Chao Huang, Yuanfeng Zou and Lixia Li from Sichuan Agricultural University for their suggestions on manuscript preparing. This study was supported by Chang Jiang Scholars Program from Chinese Ministry of Education to De Wu (T2012157). Bin Feng was a receipt of Initial Research Funding from Sichuan Agricultural University. We thank Yaohui Nie from Purdue University for reviewing the manuscript.

Author Contributions: Bin Feng, Xiaohua Huang and De Wu conceived and designed the study; Xiaohua Huang and Bin Feng performed the experiments and analyzed the data; Dandan Jiang, Lun Hua, and Yong Zhuo participated in the animal experiments; Bin Feng and Xiaohua Huang wrote the paper; De Wu reviewed and edited the paper; all authors read and approved the final manuscript.

Conflicts of Interest: The authors declare no conflict of interest.

$\begin{array}{ll}\text { Abbreviations } \\ \text { TM } & \text { Tunicamycin } \\ \text { ER } & \text { Endoplasmic reticulum } \\ \text { GRP78 } & \text { Glucose-regulated protein 78 } \\ \text { CHOP } & \text { CCAAT/enhancer-binding protein (C/EBP) homologous protein } \\ \text { PERK } & \text { Protein kinase R-like endoplasmic reticulum kinase } \\ \text { AST } & \text { Aspartate transaminase } \\ \text { ALT } & \text { Alanine transaminase } \\ \text { ApoB100 } & \text { Apolipoprotein B100 } \\ \text { ApoB } & \text { Apolipoprotein B } \\ \text { LDL-C } & \text { Low-density lipoprotein cholesterol } \\ \text { HDL-C } & \text { High-density lipoprotein cholesterol } \\ \text { FFA } & \text { Free fatty acid } \\ \text { Akt } & \text { Protein kinase B } \\ \text { GS } & \text { Glycogen synthetase } \\ \text { GP } & \text { Glycogen phosphotase } \\ \text { FAS } & \text { Fatty acid synthase } \\ \text { SCD1 } & \text { Stearoyl-CoA desaturase 1 } \\ \text { PPAR } \alpha & \text { Peroxisome proliferator-activated receptor alpha } \\ \text { CPT1a } & \text { Carnitine palmitoyltransferase 1a } \\ \text { ACADL } & \text { Acyl-CoA dehydrogenase, long chain } \\ \text { PEPCK } & \text { Phosphoenolpyruvate carboxykinase } \\ \text { G6pase } & \text { Glucose 6-phosphatase } \\ & \end{array}$




\section{References}

1. Koliaki, C.; Roden, M. Hepatic energy metabolism in human diabetes mellitus, obesity and non-alcoholic fatty liver disease. Mol. Cell Endocrinol. 2013, 379, 35-42. [CrossRef] [PubMed]

2. Oh, K.J.; Han, H.S.; Kim, M.J.; Koo, S.H. Transcriptional regulators of hepatic gluconeogenesis. Arch Pharm. Res. 2013, 36, 189-200. [CrossRef] [PubMed]

3. Hotamisligil, G.S. Endoplasmic reticulum stress and the inflammatory basis of metabolic disease. Cell 2010, 140, 900-917. [CrossRef] [PubMed]

4. Fu, S.N.; Watkins, S.M.; Hotamisligil, G.S. The Role of Endoplasmic Reticulum in Hepatic Lipid Homeostasis and Stress Signaling. Cell Metab. 2012, 15, 623-634. [CrossRef] [PubMed]

5. Araki, S.; Okazaki, M.; Goto, S. Impaired lipid metabolism in aged mice as revealed by fasting-induced expression of apolipoprotein mRNAs in the liver and changes in serum lipids. Gerontology 2004, 50, $206-215$. [CrossRef] [PubMed]

6. Postic, C.; Girard, J. Contribution of de novo fatty acid synthesis to hepatic steatosis and insulin resistance: Lessons from genetically engineered mice. J. Clin. Investig. 2008, 118, 829-838. [CrossRef] [PubMed]

7. Jensen-Urstad, A.P.; Semenkovich, C.F. Fatty acid synthase and liver triglyceride metabolism: Housekeeper or messenger? Biochim. Biophys. Acta 2012, 1821, 747-753. [CrossRef] [PubMed]

8. Choi, S.S.; Diehl, A.M. Hepatic triglyceride synthesis and nonalcoholic fatty liver disease. Curr. Opin. Lipidol. 2008, 19, 295-300. [CrossRef] [PubMed]

9. Murakami, M.; Bessho, K.; Mushiake, S.; Kondou, H.; Miyoshi, Y.; Ozono, K. Major role of apolipoprotein B in cycloheximide-induced acute hepatic steatosis in mice. Hepatol. Res. 2011, 41, 446-454. [CrossRef] [PubMed]

10. Adeva-Andany, M.M.; Gonzalez-Lucan, M.; Donapetry-Garcia, C.; Fernandez-Fernandez, C.; Ameneiros-Rodriguez, E. Glycogen metabolism in humans. BBA Clin. 2016, 5, 85-100. [CrossRef] [PubMed]

11. Boden, G. Gluconeogenesis and glycogenolysis in health and diabetes. J. Investig. Med. 2004, 52, 375-378. [CrossRef] [PubMed]

12. Bobrovnikova-Marjon, E.; Hatzivassiliou, G.; Grigoriadou, C.; Romero, M.; Cavener, D.R.; Thompson, C.B.; Diehl, J.A. PERK-dependent regulation of lipogenesis during mouse mammary gland development and adipocyte differentiation. Proc. Natl. Acad. Sci. USA 2008, 105, 16314-16319. [CrossRef] [PubMed]

13. Wang, Y.; Vera, L.; Fischer, W.H.; Montminy, M. The CREB coactivator CRTC2 links hepatic ER stress and fasting gluconeogenesis. Nature 2009, 460, 534-537. [CrossRef] [PubMed]

14. Zeng, L.; Lu, M.; Mori, K.; Luo, S.; Lee, A.S.; Zhu, Y.; Shyy, J.Y. ATF6 modulates SREBP2-mediated lipogenesis. EMBO J. 2004, 23, 950-958. [CrossRef] [PubMed]

15. Lee, A.H.; Scapa, E.F.; Cohen, D.E.; Glimcher, L.H. Regulation of hepatic lipogenesis by the transcription factor XBP1. Science 2008, 320, 1492-1496. [CrossRef] [PubMed]

16. Zhou, Y.; Lee, J.; Reno, C.M.; Sun, C.; Park, S.W.; Chung, J.; Lee, J.; Fisher, S.J.; White, M.F.; Biddinger, S.B.; Ozcan, U. Regulation of glucose homeostasis through a XBP-1-FoxO1 interaction. Nat. Med. 2011, 17, 356-365. [CrossRef] [PubMed]

17. Zhang, Y.; Liao, S.; Fan, W.; Wei, W.; Wang, C.; Sun, S. Tunicamycin-induced ER stress regulates chemokine CCL5 expression and secretion via STAT3 followed by decreased transmigration of MCF-7 breast cancer cells. Oncol. Rep. 2014, 32, 2769-2776. [CrossRef] [PubMed]

18. Yen, J.H.; Wu, P.S.; Chen, S.F.; Wu, M.J. Fisetin Protects PC12 Cells from Tunicamycin-Mediated Cell Death via Reactive Oxygen Species Scavenging and Modulation of Nrf2-Driven Gene Expression, SIRT1 and MAPK Signaling in PC12 Cells. Int. J. Mol. Sci. 2017, 18, 852. [CrossRef] [PubMed]

19. Banerjee, A.; Lang, J.Y.; Hung, M.C.; Sengupta, K.; Banerjee, S.K.; Baksi, K.; Banerjee, D.K. Unfolded protein response is required in $n u / n u$ mice microvasculature for treating breast tumor with tunicamycin. J. Biol. Chem. 2011, 286, 29127-29138. [CrossRef] [PubMed]

20. Han, X.; Zhang, X.; Li, H.; Huang, S.; Zhang, S.; Wang, F.; Shi, Y. Tunicamycin enhances the antitumor activity of trastuzumab on breast cancer in vitro and in vivo. Oncotarget 2015, 6, 38912-38925. [CrossRef] [PubMed]

21. Hou, H.; Sun, H.; Lu, P.; Ge, C.; Zhang, L.; Li, H.; Zhao, F.; Tian, H.; Chen, T.; Yao, M.; Li, J. Tunicamycin potentiates cisplatin anticancer efficacy through the DPAGT1/Akt/ABCG2 pathway in mouse Xenograft models of human hepatocellular carcinoma. Mol. Cancer Ther. 2013, 12, 2874-2884. [CrossRef] [PubMed] 
22. Whiteman, E.L.; Cho, H.; Birnbaum, M.J. Role of Akt/protein kinase B in metabolism. Trends Endocrinol. Metab. 2002, 13, 444-451. [CrossRef]

23. Jung, T.W.; Hwang, H.J.; Hong, H.C.; Choi, H.Y.; Yoo, H.J.; Baik, S.H.; Choi, K.M. Resolvin D1 reduces ER stress-induced apoptosis and triglyceride accumulation through JNK pathway in HepG2 cells. Mol. Cell. Endocrinol. 2014, 391, 30-40. [CrossRef] [PubMed]

24. Chang, Y.S.; Tsai, C.T.; Huangfu, C.A.; Huang, W.Y.; Lei, H.Y.; Lin, C.F.; Su, I.J.; Chang, W.T.; Wu, P.H.; Chen, Y.T.; et al. ACSL3 and GSK-3 $\beta$ are essential for lipid upregulation induced by endoplasmic reticulum stress in liver cells. J. Cell. Biochem. 2011, 112, 881-893. [CrossRef] [PubMed]

25. Oyadomari, S.; Mori, M. Roles of CHOP/GADD153 in endoplasmic reticulum stress. Cell Death Differ. 2004, 11, 381-389. [CrossRef] [PubMed]

26. Lhotak, S.; Sood, S.; Brimble, E.; Carlisle, R.E.; Colgan, S.M.; Mazzetti, A.; Dickhout, J.G.; Ingram, A.J.; Austin, R.C. ER stress contributes to renal proximal tubule injury by increasing SREBP-2-mediated lipid accumulation and apoptotic cell death. Am. J. Physiol. Renal. Physiol. 2012, 303, F266-F278. [CrossRef] [PubMed]

27. AbouRjaili, G.; Shtaynberg, N.; Wetz, R.; Costantino, T.; Abela, G.S. Current concepts in triglyceride metabolism, pathophysiology, and treatment. Metabolism 2010, 59, 1210-1220. [CrossRef] [PubMed]

28. Ishikawa, M.; Yoshida, K.; Okamura, H.; Ochiai, K.; Takamura, H.; Fujiwara, N.; Ozaki, K. Oral Porphyromonas gingivalis translocates to the liver and regulates hepatic glycogen synthesis through the Akt/GSK-3beta signaling pathway. Biochim. Biophys. Acta 2013, 1832, 2035-2043. [CrossRef] [PubMed]

29. Ozcan, U.; Yilmaz, E.; Ozcan, L.; Furuhashi, M.; Vaillancourt, E.; Smith, R.O.; Gorgun, C.Z.; Hotamisligil, G.S. Chemical chaperones reduce ER stress and restore glucose homeostasis in a mouse model of type 2 diabetes. Science 2006, 313, 1137-1140. [CrossRef] [PubMed]

30. Lee, J.S.; Zheng, Z.; Mendez, R.; Ha, S.W.; Xie, Y.; Zhang, K. Pharmacologic ER stress induces non-alcoholic steatohepatitis in an animal model. Toxicol. Lett. 2012, 211, 29-38. [CrossRef] [PubMed]

31. Lee, S.Y.; Hong, I.K.; Kim, B.R.; Shim, S.M.; Sung Lee, J.; Lee, H.Y.; Soo Choi, C.; Kim, B.K.; Park, T.S. Activation of sphingosine kinase 2 by endoplasmic reticulum stress ameliorates hepatic steatosis and insulin resistance in mice. Hepatology 2015, 62, 135-146. [CrossRef] [PubMed]

32. Wu, F.L.; Liu, W.Y.; Van Poucke, S.; Braddock, M.; Jin, W.M.; Xiao, J.; Li, X.K.; Zheng, M.H. Targeting endoplasmic reticulum stress in liver disease. Expert. Rev. Gastroenterol. Hepatol. 2016, 10, 1041-1052. [CrossRef] [PubMed]

33. Petta, S.; Gastaldelli, A.; Rebelos, E.; Bugianesi, E.; Messa, P.; Miele, L.; Svegliati-Baroni, G.; Valenti, L.; Bonino, F. Pathophysiology of Non Alcoholic Fatty Liver Disease. Int. J. Mol. Sci. 2016, 17, 2082. [CrossRef] [PubMed]

34. Ono, H.; Shimano, H.; Katagiri, H.; Yahagi, N.; Sakoda, H.; Onishi, Y.; Anai, M.; Ogihara, T.; Fujishiro, M.; Viana, A.Y.; et al. Hepatic Akt activation induces marked hypoglycemia, hepatomegaly, and hypertriglyceridemia with sterol regulatory element binding protein involvement. Diabetes 2003, 52, 2905-2913. [CrossRef] [PubMed]

35. Cohen, D.E.; Fisher, E.A. Lipoprotein metabolism, dyslipidemia, and nonalcoholic fatty liver disease. Semin. Liver Dis. 2013, 33, 380-388. [PubMed]

36. Ros, S.; Zafra, D.; Valles-Ortega, J.; Garcia-Rocha, M.; Forrow, S.; Dominguez, J.; Calbo, J.; Guinovart, J.J. Hepatic overexpression of a constitutively active form of liver glycogen synthase improves glucose homeostasis. J. Biol. Chem. 2010, 285, 37170-37177. [CrossRef] [PubMed]

37. Feng, B.; Jiao, P.; Helou, Y.; Li, Y.; He, Q.; Walters, M.S.; Salomon, A.; Xu, H. Mitogen-activated protein kinase phosphatase 3 (MKP-3)-deficient mice are resistant to diet-induced obesity. Diabetes 2014, 63, 2924-2934. [CrossRef] [PubMed]

38. Feng, B.; He, Q.; Xu, H. FOXO1-dependent up-regulation of MAP kinase phosphatase 3 (MKP-3) mediates glucocorticoid-induced hepatic lipid accumulation in mice. Mol. Cell. Endocrinol. 2014, 393, 46-55. [CrossRef] [PubMed]

(C) 2017 by the authors. Licensee MDPI, Basel, Switzerland. This article is an open access article distributed under the terms and conditions of the Creative Commons Attribution (CC BY) license (http:/ / creativecommons.org/licenses/by/4.0/). 